\title{
Aerodynamic heating of ballistic missile including the effects of gravity
}

\author{
S N MAITRA \\ Mathematics Department, National Defence Academy, Khadakwasla, \\ Pune 411023 , India
}

MS received on 1 February 1999

\begin{abstract}
The aerodynamic heating of a ballistic missile due to only convection is analysed taking into consideration the effects of gravity. The amount of heat transferred to the wetted area and to the nose region has been separately determined, unlike A Miele's treatise without consideration of gravity. The peak heating rates to the wetted area and to the nose of the missile are also investigated. Finally four numerical examples are cited to estimate the errors, in heat transfers and heating rates to both wetted area and nose region of the missile, arising out of neglecting the gravitational forces.
\end{abstract}

Keywords. Aerodynamic heating; ballistic missile; gravity; flat-earth.

\section{Introduction}

In previous work (Miele 1962; Maitra 1979) the re-entry of ballistic missiles for low and high ballistic factors was analysed within the framework of the flat-earth hypothesis, taking into consideration the effect of the weight component of the missile on the tangent to the flight path, and also the changes in the path inclination espoused by the gravitational force. Having retained these viewpoints in this paper, the aerodynamic heating of the missile is studied while Miele (1962) developed aerodynamic heating criteria disregarding the effects of gravity and variation of path inclination caused by gravity itself as he speculated that the gravitational force could entail only small deviations in the missile velocity-distribution. As is done by him, in this paper also, only convective heating is dealt with because radiant heat is negligible with respect to the former. However, velocity distribution plays the main role in convective heating of ballistic missiles.

R D Neumann and J R Hayes (Summerfield 1986) carried out a sort of aerodynamic heating analysis for high velocity missiles by applying the knowledge of both inviscid and viscous flow fields over the vehicle, as the heating rate at any point of the vehicle is a function of many test-specific properties such as fluid flow field stagnation conditions, fluid temperature, boundary-layer state, vehicle attitude, viscous effects etc.

A list of symbols is given at the end of the paper 


\section{Overall heat transfer to the wetted area}

Computation of heating rates is necessary in the structural design of any space vehicle. Rate of convective heat transfer to the wetted area, based on Miele's (1962) hypothesis, is rewritten as:

$$
\dot{Q}=\frac{1}{4} C_{F} \rho S_{W} V^{3}
$$

The deceleration equation (Miele 1962; Maitra 1979) involving the aerodynamic drag and gravitational force is

$$
\dot{V}=-((D / W)+\sin \theta) g .
$$

Combining (1) and (2), replacing the path indication $\theta$ by its initial value $\theta_{i}$, because of its small variation, using equations (6) and (7) from Maitra (1979) and then simplifying, we get the heat transfer per unit velocity variation as

$$
\frac{\mathrm{d} Q}{\mathrm{~d} V}=\frac{-C_{F} \rho S_{W} V^{3}}{4 g}\left(\frac{C_{D} \rho S V^{2}}{2 W}+\sin \theta_{i}\right)^{-1}=\frac{-W C_{F} S_{W} V^{3}}{2 g C_{D} S}\left(\frac{V^{2}+2 W \sin \theta_{i}}{C_{D} \rho S}\right)^{-1}
$$

or

$$
\frac{\mathrm{d} Q}{\mathrm{~d} V}=\frac{-W C_{F} S_{W}}{2 g C_{D} S}\left[V-\frac{2\left\{\left(W \sin \theta_{i}\right) /\left(C_{D} \rho S\right)\right\} V}{V^{2}+2\left(W \sin \theta_{i}\right) /\left(C_{D} \rho S\right)}\right] .
$$

Now integrating (3) from the initial to the final values one gets

$$
\begin{aligned}
& V=V_{i} \quad \text { and } \quad Q=Q_{i}=0 \\
& V=V_{f} \quad \text { and } \quad Q=Q_{f} \\
& Q=\frac{W C_{F} S_{W}}{4 g C_{D} S}\left[\left(V_{i}^{2}-V_{f}^{2}\right)+2 \int_{V_{i}}^{V_{f}} \frac{\left\{2 V\left(W \sin \theta_{i}\right) /\left(C_{D} \rho S\right)\right\} \mathrm{d} V}{V^{2}+2\left(W \sin \theta_{i}\right) /\left(C_{D} \rho\right)}\right]=Q_{1}+Q_{2} .
\end{aligned}
$$

It is observed that the first term $Q_{1}$ on the right hand side of (4) gives the expression for the heat transfer due to consideration of the motion excluding the effects of gravity and the second term $Q_{2}$ stands for the addition to the heat transfer brought about by the gravity. It would be convenient to evaluate this integral if the variables are expressed in nondimensional forms (Miele 1962; Maitra 1979) recalled as

$$
\begin{aligned}
\eta & =h / \lambda, \quad u=V / \sqrt{\lambda g}, \\
K_{B} & =-K_{D} / \sin \theta_{i}=-C_{D} \rho_{0} S \lambda g /\left(2 W \sin \theta_{i}\right), \\
\pi & =2 K_{B} \rho / \rho_{0}=2 K_{B} e^{-\eta}, \\
u / \sqrt{2} & =\left\{C+E_{i}(\pi)\right\}^{1 / 2} e^{-\pi / 2},
\end{aligned}
$$

so that with the initial conditions $\pi=\pi_{i}, u=u_{i}, \eta=\eta_{i}$, and the final conditions $\pi=\pi_{f}$, $u=u_{f}, \eta=\eta_{f}$,

$$
Q_{2}^{\prime}=2 \lambda g \int_{\pi_{i}}^{\pi_{f}} \frac{2 u \mathrm{~d} u}{\pi\left(u^{2}-2 / \pi\right)}
$$




$$
=-2 \lambda g \int_{\pi_{i}}^{\pi_{f}} \frac{\mathrm{d}\left(u^{2}\right)}{u^{2} \pi-2}=-2 \lambda g \int_{\pi_{i}}^{\pi_{f}} \frac{\mathrm{d}\left(u^{2} / 2\right)}{\pi\left(\frac{u^{2}}{2}-\frac{1}{\pi}\right)} .
$$

On using equation (4) from Miele (1962, p. 298) or, equivalently, equation (10) from Maitra (1979) in the above integral, it reduces to the simple form

$$
Q_{2}^{\prime}=2 \lambda g \int_{\pi_{i}}^{\pi_{f}} \frac{\mathrm{d} \pi}{\pi}=2 \lambda g \log \frac{\pi_{f}}{\pi_{i}}>0
$$

Therefore, (4) gives the total heat transfer at any time $t$ as

$$
Q=\frac{W C_{F} S_{W} \lambda}{4 C_{D} S}\left(u_{i}^{2}-u_{f}^{2}+2 \log \frac{\pi_{f}}{\pi_{i}}\right)
$$

where $u_{i}, u_{f}, \pi_{i}, \pi_{f}$ are to be determined from (5). After introducing ballistic frictional factor and reference energy (Miele 1962) to (6), such that

$$
\begin{aligned}
K_{B F} & =-C_{F} \rho_{0} S_{W} \lambda g /\left(2 W \sin \theta_{i}\right), \\
Q_{R} & =W V_{i}^{2} /(2 g),
\end{aligned}
$$

the fraction of the initial kinetic energy transferred to the missile wetted-area in the form of heat can be obtained as

$$
\frac{Q}{Q_{R}}=\frac{1}{2} \frac{K_{B F}}{K_{B}}\left\{1-\left(u_{f}^{2}-2 \log \frac{\pi_{f}}{\pi_{i}}\right) / u_{i}^{2}\right\}
$$

which by use of the last of set (5) can be rewritten as

$$
\frac{Q}{Q_{R}}=\frac{K_{B F}}{2 K_{B}}\left[1-\left\{C+E_{i}\left(\pi_{i}\right)\right\}^{-1}\left\{C+E_{i}\left(\pi_{f}\right)-\left(\log \frac{\pi_{f}}{\pi_{i}}\right) e^{\pi_{f}}\right\} e^{-\left(\pi_{f}-\pi_{i}\right)}\right] .
$$

Now $\pi=\pi_{f}=2 K_{B}$ at sea level where $\eta=0$ vide the first of (5). Hence the foregoing fraction of the kinetic energy in the form of heat, when the missile strikes the surface at sea level, becomes

$$
\begin{aligned}
\frac{Q_{f}}{Q_{R}}= & \frac{1}{2} \frac{K_{B F}}{K_{B}}\left[1-\left\{C+E_{i}\left(\pi_{i}\right)\right\}^{-1}\left\{C+E_{i}\left(2 K_{B}\right)-\eta_{i} e^{2 K_{B}}\right\} e^{-2 K_{B}+\pi_{i}}\right] \\
& \times\left(\log \frac{\pi_{f}}{\pi_{i}}=\eta_{i}-\eta_{f}\right) .
\end{aligned}
$$

Further with values (Miele 1962, Maitra 1979) of $C$ between 150 and 350, the re-entry altitude $h_{i}=200,000 \mathrm{ft}$ or $60937 \mathrm{~m}, \pi_{i}$ and $E_{i}\left(\pi_{i}\right)$ both become too small to be included in (9) so that it turns out to be

$$
\frac{Q_{f}}{Q_{R}}=\frac{K_{B F}}{2 K_{B}}\left[1-e^{-2 K_{B}}-\frac{1}{C}\left\{E_{i}\left(2 K_{B}\right) e^{-2 K_{B}}-\eta_{i}\right\}\right] .
$$

On comparison of (10) with (19) of Miele (1962), an additional heat-transfer term is seen due to the effects of gravity. Following the same trends as in Miele's (1962) analysis, in the gravity-included case we ascertain for relatively light ballistic missile $\left(K_{B} \gg 1\right)$ whether 
$e^{-2 K_{B}} \simeq 0$ and $E_{i}\left(2 K_{B}\right) e^{-2 K_{B}} \ll 1$ so that (10) reduces to the form

$$
\frac{Q_{f}}{Q_{R}}=\frac{K_{B F}}{2 K_{B}}\left(1+\frac{\eta_{i}}{C}\right) .
$$

Similarly for a relatively heavy ballistic missile $\left(K_{B} \ll 1\right)$

$$
e^{-2 K_{B}} \simeq 1-2 K_{B}
$$

whence (10) takes up the form

$$
\frac{Q_{f}}{Q_{R}}=K_{B F}\left[1-\frac{1}{2 C}\left\{\left(\frac{1}{K_{B}}-2\right) E_{i}\left(2 K_{B}\right)-\frac{\eta_{i}}{K_{B}}\right\}\right] .
$$

Comparison of equations (20) and (22) from Miele (1962) with (11) and (12) of the present analysis reveals that the ratios of the above heat energies with inclusion of gravity to that without its inclusion, in the cases of relatively light and heavy missiles respectively, are

$$
\left(C+\eta_{i}\right): C
$$

and

$$
\left\{2 C K_{B}-\left(1-2 K_{B}\right) E_{i}\left(2 K_{B}\right)+\eta_{i}\right\}: 2 C K_{B} .
$$

It is, therefore, evident that the effects of gravity help increase the heat transfer to the ballistic missile to some extent. This additional heat-transfer term arising out of consideration of the gravitational effects is not insignificant from the viewpoint of more accurate design of the missile or its perfect design.

Introducing the reference heating rate as (Miele 1962, p. 318)

$$
\dot{Q}_{R}=\frac{-W}{4 K g} \cdot \frac{K_{B F}}{K_{B}} V_{i}^{3} \sin \theta_{i},
$$

and with the help of the last of (5) in combination with (1), the non-dimensional heating rate to the wetted area is given by

$$
\dot{Q} / \dot{Q}_{R}=\pi\left\{\left(C+E_{i}(\pi)\right)\left(C+E_{i}\left(\pi_{i}\right)\right)^{-1}\right\}^{3 / 2} e^{-3 \pi / 2},
$$

which attains an analytical maximum at the altitude at which $\pi$ is given by

$$
(\pi-(2 / 3))\left\{C+E_{i}(\pi)\right\}-e^{\pi}=0,
$$

subject to the condition

$$
2\left(K_{B}-(1 / 3)\right)\left\{C+E_{i}\left(2 K_{B}\right)\right\}-e^{2 K_{B}}>0,
$$

such that

$$
\left(\dot{Q} / \dot{Q}_{R}\right)_{\max }=\pi\left(\pi-\frac{2}{3}\right)^{-3 / 2}\left\{C+E_{i}\left(\pi_{i}\right)\right\}^{-3 / 2},
$$

and from (15a) and (5) the corresponding velocity is given by

$$
u=2^{1 / 2}(\pi-(2 / 3))^{-1 / 2} \text {. }
$$


On the other hand, if $K_{B}<(1 / 3)$, then the maximum heating rate, not an analytical maximum, that occurs at sea level becomes

$$
\dot{Q} / \dot{Q}_{R}=2 K_{B}\left\{C+E_{i}\left(2 K_{B}\right)\right\}^{3 / 2}\left\{C+E_{i}\left(\pi_{i}\right)\right\}^{-3 / 2} e^{-3 K_{B}} .
$$

Hence, for ballistic factor larger than $1 / 3$, i.e. for relatively light ballistic missiles the peak heating in view of (14) and (8) approximately satisfies the same relationship (Miele 1962)

$$
\dot{Q} \simeq K_{B F} / K_{B}
$$

and if the missile is relatively heavy, i.e. the ballistic factor $K_{B} \ll 1$ the previous relationship can be replaced by

$$
\dot{Q} \simeq K_{B F}\left[1+\left(3 \log \left(2 K_{B}\right) / 2 C\right)\right] .
$$

Heating to the nose: According to the hypotheses (Miele 1962) the rate of heat transfer at either stagnation (laminar flow) or sonic (turbulent flow) of the nose region can be represented by

$$
\dot{q}=C \rho^{x} V^{y} / \gamma^{z},
$$

whereas the relevant reference heating rate is

$$
\dot{q}_{R}=C\left(\rho_{0} / 2 K_{B}\right)^{x}\left(V_{i}^{y} / \gamma^{z}\right) .
$$

Combining (21) and (22), we get in nondimensional form

$$
\dot{q} / \dot{q}_{R}=\pi^{x}\left\{C+E_{i}(\pi)\right\}^{y / 2}\left\{C+E_{i}\left(\pi_{i}\right)\right\}^{-y / 2} e^{-\pi y / 2},
$$

which shows that the heating rate has a stationary point. Taking the logarithm of both sides of (23), differentiating with respect to $\pi$ and then equating to zero, one can have the analytical maximum of the heating rate given by

$$
\left(\frac{\dot{q}}{\dot{q}_{R}}\right)_{\max }=\pi^{x}\left\{\frac{y}{(\pi y-2 x)\left(C+E_{i}\left(\pi_{i}\right)\right)}\right\}^{y / 2},
$$

where

$$
-(\pi y-2 x)\left\{C+E_{i}(\pi)\right\}+e^{\pi} y=0
$$

subject to

$$
2\left(x-K_{B} y\right)\left\{C+E_{i}\left(2 K_{B}\right)\right\}+y e^{2 K_{B}}>0,
$$

Further, the time rate of $\pi$ is rewritten as

$$
\frac{\mathrm{d} \pi}{\mathrm{d} t}=-\left(\frac{2 g}{\lambda}\right)^{1 / 2} \pi e^{-\pi / 2}\left\{C+E_{i}(\pi)\right\}^{1 / 2} \sin \theta_{i} .
$$

Combination of (23) and (25) leads to

$$
\frac{1}{\dot{q}_{R}} \cdot \frac{\mathrm{d} q}{\mathrm{~d} \pi}=-u_{i}^{-y}\left(\sin \theta_{i}\right)^{-1}(\lambda / g)^{1 / 2}\left\{C+E_{i}(\pi)\right\}^{(y-1) / 2} e^{-(y-1) \pi / 2} \pi^{x-1},
$$


on integrating which, from initial to final values, total heat transfer to either of the two critical points of the nose is given by

$$
q=\frac{-\dot{q}_{R}}{u_{i}^{y} \sin \theta_{i}}\left(\frac{\lambda}{g}\right)^{1 / 2} \int_{\pi_{i}}^{\pi_{f}}\left\{C+E_{i}(\pi)\right\}^{y-1 / 2} e^{-(y-1 / 2) \pi} \pi^{x-1} \mathrm{~d} x
$$

or

$$
q=-\left\{\dot{q}_{R} I /\left(u_{i}^{y} \sin \theta_{i}\right)\right\}\left(\frac{\lambda}{g}\right)^{1 / 2}
$$

where

$$
\begin{gathered}
m=(y-1) / 2, \quad n=x-1 \quad \text { and } \\
I=\int_{\pi_{i}}^{\pi_{f}}\left\{C+E_{i}(\pi)\right\}^{m} e^{-m \pi} \pi^{n} \mathrm{~d} x .
\end{gathered}
$$

Integral $I$ is in general to be evaluated numerically especially when the ballistic factor $K_{B}$ is too high to have $E_{i}(\pi)$ not much less than $C$ or $E_{i}(\pi)>C$. But for moderately low ballistic factors, e.g., $K_{B} \leq 2.5$ with positive integral values of $m$ and $n$, and $150 \leq$ $C \leq 350$ the integral can be evaluated in closed form with desired accuracy in the following manner.

Expanding the integrand of (27) binomially and neglecting the squares and other higher powers of $E_{i}(\pi)$ we can express the integral as

$$
I=C^{m} \int_{\pi_{i}}^{\pi_{f}}\left\{1+\frac{m E_{i}(\pi)}{C}\right\} e^{-m \pi} \pi^{n} \mathrm{~d} \pi,
$$

or

$$
I=C^{m} I_{m, n}+m C^{m-1} E_{m, n},
$$

where

$$
\begin{aligned}
I_{m, n}= & \int_{\pi_{i}}^{\pi_{f}} e^{-m \pi} \pi^{n} \mathrm{~d} \pi=\frac{\pi_{i}^{n} e^{-m \pi_{i}}-\pi_{f} e^{-m \pi_{f}}}{m}+\frac{n}{m} I_{m, n-1}, \\
I_{m, 0}= & \left(e^{-\pi_{i}}-e^{-\pi_{f}}\right) / m, \\
E_{m, n}= & \int_{\pi_{i}}^{\pi_{f}} E_{i}(\pi) e^{-m \pi} \pi^{n} \mathrm{~d} \pi=\left\{E_{i}\left(\pi_{i}\right) \pi_{i}^{n} e^{-m \pi_{i}}-E_{i}\left(\pi_{f}\right) \pi_{f}^{n} e^{-m \pi_{f}}\right\} / m \\
& +\frac{1}{m} \int_{\pi_{i}}^{\pi_{f}} e^{-m \pi}\left\{e^{\pi} \pi^{n-1}+E_{i}(\pi) n \pi^{n-1}\right\} \mathrm{d} \pi,
\end{aligned}
$$

or

$$
\begin{aligned}
& E_{m, n}=\left\{E_{i}\left(\pi_{i}\right) \pi_{i}^{n} e^{-n \pi_{i}}-E_{i}\left(\pi_{f}\right) \pi_{f}^{n} e^{-m \pi_{f}}\right\} / m+I_{m-1, n-1} / m+\frac{n}{m} E_{m, n-1}, \\
& E_{m, 0}=\int_{\pi_{i}}^{\pi_{f}} E_{i}(\pi) e^{-m \pi} \mathrm{d} \pi=\left\{E_{i}\left(\pi_{i}\right) e^{-m \pi_{i}}-E_{i}\left(\pi_{f}\right)\right\} / m+\frac{1}{m} \int_{\pi_{i}}^{\pi_{f}} \frac{e^{-(m-1) \pi} \mathrm{d} \pi}{\pi}
\end{aligned}
$$


or

$$
\begin{aligned}
E_{m, 0}=\{ & \left.E_{i}\left(\pi_{i}\right) e^{-m \pi_{i}}-E_{i}\left(\pi_{f}\right) e^{-m \pi_{f}}\right\} / m+\frac{1}{m}\left[E_{i}\left\{-(m-1) \pi_{f}\right\}\right. \\
& \left.-E_{i}\left\{-(m-1) \pi_{i}\right\}\right] .
\end{aligned}
$$

Thus we see that heat transfer to the two critical points of the nose, i.e., the integral (26) can be evaluated in closed form by use of reduction process as evolved in (28) to (31).

Equation (24b) shows that for relatively light ballistic missiles, i.e., with the ballistic factor $K_{B} \gg x / y$ and for relatively heavy ballistic factor, i.e., with the ballistic factor $K_{B} \ll x / y$, the peak heating rate to the nose follows, in the gravity-included case, more or less the same trends as in the gravity-excluded case (Miele 1962, pp 321-323). Further it can be added that in consequence of (5) and (23), the peak heating rate, not an analytical maximum, occurs at sea level, given by

$$
\frac{\dot{q}}{\dot{q}_{R}}=\left(2 K_{B}\right)^{x}\left\{C+E_{i}\left(2 K_{B}\right)\right\}^{y / 2}\left\{C+E_{i}\left(\pi_{i}\right)\right\}^{-y / 2} e^{-2 K_{B} y},
$$

subject to the constraint

$$
2\left(x-K_{B} y\right)\left\{C+E_{i}\left(2 K_{B}\right)\right\}+y e^{2 K_{B}}<0 .
$$

Numerical example 1: First let us consider a long range ballistic missile with ballistic factor $K_{B}=5$, dimensionless initial velocity $u_{i}=24$, initial altitude $\eta_{i}=5$ that leads to $\pi_{i}=0.003$. From figure 6 of Miele (1962, p. 295) the final velocity of the missile on striking the earth's surface $\left(\eta_{f}=0\right)$, where $\pi_{f}=2 K_{B}$, is obtained as $u_{f}=5$ in the gravity-included case. Hence by use of (6) the percentage of error due to neglecting gravity turns out to be

$$
\frac{2 \times 100 \log \left(\pi_{f} / \pi_{i}\right)}{u_{i}^{2}-u_{f}^{2}}=\frac{\left(\eta_{i}-\eta_{f}\right) \times 200}{u_{i}^{2}-u_{f}^{2}}=\frac{(5-0) \times 200}{576-225}=2.8 \% .
$$

For a ballistic missile, $K_{B}=5$ and $u_{i}=25$, leading to $u_{f}=0.15$; the other constant parameters being the same as in the previous case, the error percentage in the heat transfer to the wetted area for neglecting the gravity amounts to

$$
\epsilon=\frac{2 \times(5-0) \times 100}{25^{2}-(0.15)^{2}}=1.6 \% \text {. }
$$

The positive value in both the cases indicates that the heat transfer to the wetted area in fact increases due to the effects of gravity.

Numerical example 2: If we choose $K_{B}=1, \eta_{i}=10, C=165$ for a short range ballistic missile, then $E_{i}\left(\pi_{i}\right)=0.001$ and on account of (15a) and (16) the maximum heating rate to the wetted area occurs at $\pi=0.6788$, i.e., at the non-dimensional altitude $\eta=1.08$ and is given by

$$
\dot{Q} / \dot{Q}_{R}=0.2480
$$

whereas neglecting the gravity (Miele 1962, p. 27) this value works out to be

$$
\dot{Q} / \dot{Q}_{R}=0.2462
$$


Table 1. Flow related index parameters.

\begin{tabular}{lcccc}
\hline & $C$ & $x$ & $y$ & $z$ \\
\hline $\begin{array}{c}\text { Stagnation point } \\
\text { (laminar flow) }\end{array}$ & $1.55 \times 10^{-5}$ & 0.5 & 3 & 0.5 \\
$\begin{array}{c}\text { Sonic point } \\
\text { (turbulent flow) }\end{array}$ & $7.45 \times 10^{-4}$ & 0.8 & 3 & 0.2 \\
\hline
\end{tabular}

and the corresponding altitude is (Miele 1962)

$$
\eta=\log \left(3 K_{B}\right)=\log 3=1.099 .
$$

Now let us take $K_{B}<1 / 3$ say, $K_{B}=0.025$ and $C=150$, then the peak heating rate occurs at sea level and by use of (18) can be computed as

$$
\left(\dot{Q} / \dot{Q}_{R}\right)_{\max }=0.2414
$$

while disregarding gravity (Miele 1962, p. 27) the same gives

$$
\left(\dot{Q} / \dot{Q}_{R}\right)_{\max }=0.2354
$$

Numerical example 3: From table 1 and (31) the heat transfer at the stagnation point of the nose reduces to the form

$$
q=\frac{-\dot{q}_{R}}{u_{i}^{3} \sin \theta_{i}}\left(\frac{\lambda}{g}\right)^{1 / 2} \int_{\pi_{i}}^{\pi_{f}}\{C+E(\pi)\} e^{-\pi} \pi^{-1 / 2} \mathrm{~d} \pi
$$

For low ballistic factor $K_{B} \ll 1, \pi_{i}<\pi<\pi_{f} \ll 1$, so that $e^{-\pi} \simeq 1-\pi$ and as such

$$
\int_{\pi_{i}}^{\pi_{f}}\left\{C+E_{i}(\pi)\right\} e^{-\pi} \pi^{-1 / 2} \mathrm{~d} \pi=\int_{\pi_{i}}^{\pi_{f}}\left\{C+E_{i}(\pi)\right\}(1-\pi) \pi^{-1 / 2} \mathrm{~d} \pi
$$

(which integrating by parts)

$$
=2\left[\left(\pi^{1 / 2}-\pi^{3 / 2} / 3\right)\left\{C+E_{i}(\pi)\right\}\right]_{\pi_{i}}^{\pi_{f}}-2 \int_{\pi_{i}}^{\pi_{f}}\left(\pi^{1 / 2}-\frac{\pi^{3 / 2}}{3}\right) \frac{e^{\pi}}{\pi} \mathrm{d} \pi
$$

(using once again $e^{\pi}=1+\pi$ and then further integrating)

$$
\begin{aligned}
= & 2\left[\left(\pi_{f}^{1 / 2}-\frac{\pi_{f}^{3 / 2}}{3}\right)\left\{C+E_{i}\left(\pi_{f}\right)\right\}-\left(\pi_{i}^{1 / 2}-\frac{\pi_{i}^{3 / 2}}{3}\right)\left\{C+E_{i}\left(\pi_{i}\right)\right\}\right] \\
& -4\left[\left(\pi_{f}^{1 / 2}-\pi_{i}^{1 / 2}\right)+\frac{2}{9}\left(\pi_{f}^{3 / 2}-\pi_{i}^{3 / 2}\right)-\frac{1}{15}\left(\pi_{f}^{5 / 2}-\pi_{i}^{5 / 2}\right)\right] .
\end{aligned}
$$

But the same integral associated with the non-gravity situation is

$$
\begin{aligned}
C \int_{\pi_{i}}^{\pi_{f}} e^{-\pi} \pi^{-1 / 2} \mathrm{~d} \pi & =C \int_{\pi_{i}}^{\pi_{f}}(1-\pi) \pi^{-1 / 2} \mathrm{~d} \pi \\
& =2 C\left\{\left(\pi_{f}^{1 / 2}-\pi_{i}^{1 / 2}\right)-\left(\pi_{f}^{3 / 2}-\pi_{i}^{3 / 2}\right) / 3\right\}
\end{aligned}
$$


if $K_{B}=0.3, \pi_{i}=0.001$, giving the value of $C$ equal to 200, then by use of (32) to (34) the error, owing to noninclusion of gravity in the heat transfer at the stagnation point (laminar flow) to the nose at sea level, turns out to be $0.005 \%$. However, for moderately large or very large ballistic factors this error will be much higher by virtue of (32) to (34). Further, it is interesting to calculate from (26) the heat flow to the sonic point (turbulent flow) of the nose where $x=1$,

$$
q=\frac{-\dot{q}_{R}}{u_{i}^{3} \sin \theta_{i}}\left(\frac{\lambda}{g}\right)^{1 / 2} \int_{\pi_{i}}^{\pi_{f}}\left\{C+E_{i}(\pi)\right\} e^{-\pi} \mathrm{d} \pi, \quad(y=3) .
$$

Integrating by parts and considering $C+E_{i}(\pi)$ as the first form and remembering

$$
\begin{aligned}
& \frac{\mathrm{d}}{\mathrm{d} \pi}\left\{C+E_{i}(\pi)\right\}=e^{\pi} / \pi, \\
& q=\frac{\dot{q}_{R}}{u_{i}^{3} \sin \theta_{i}}\left(\frac{\lambda}{g}\right)^{1 / 2}\left[-\left.e^{-\pi}\left\{C+E_{i}(\pi)\right\}\right|_{\pi_{i}} ^{\pi_{f}}+\int_{\pi_{i}}^{\pi_{f}} \frac{\mathrm{d} \pi}{\pi}\right],
\end{aligned}
$$

or

$$
q=\frac{-\dot{q}_{R}}{u^{3} \sin \theta_{i}}\left(\frac{\lambda}{g}\right)^{1 / 2}\left[\left\{C+E_{i}(\pi)\right\} e^{-\pi_{i}}-\left\{C+E_{i}\left(\pi_{f}\right)\right\} e^{-\pi_{f}}+\log \frac{\pi_{f}}{\pi_{i}}\right] .
$$

However, the heat flow under the same conditions concerned with (36) but without taking into account the gravity amounts to

$$
\begin{aligned}
q & =\frac{\dot{q}_{R}}{u_{i}^{3} \sin \theta_{i}}\left(\frac{\lambda}{g}\right)^{1 / 2} C \int_{\pi_{i}}^{\pi_{f}} e^{-\pi} \mathrm{d} \pi \\
& =\frac{-\dot{q}_{R}}{u_{i}^{3} \sin \theta_{i}}\left(\frac{\lambda}{g}\right)^{1 / 2} C\left(e^{-\pi_{i}}-e^{-\pi_{f}}\right) .
\end{aligned}
$$

If we take $K_{B}=1, C=200$ and $\pi_{i}=0.001$ in view of (36) and (37), we observe the heat transfer at the sonic point of the nose to have an error of $0.01 \%$ owing to neglecting the gravity. This error factor increases with the increase in ballistic factor.

Numerical example 4: With regard to table 1 , for $K_{B}=3, C=150$ and $\pi=0.001$, the heating rate at the stagnation point of the nose has in consequence of (23) and (24b) a maximum at $\pi=\frac{1}{3}+\frac{7}{10^{3}}$ i.e., $\eta=1.1$ with $9 \%$ increase in the gravity-included case. It is surmised that this maximum rate of heating, when calculated separately with and without the effects of gravity, occurs almost at the same altitude but with considerably different magnitudes. But if we take $K_{B}=0.2<1 / 3$ keeping the other constant parameters same, the heating rate at the sonic point of the nose takes place at sea level and exhibits an increase of $7.3 \%$ over the value calculated without gravitational effects.

\section{Conclusion}

The foregoing analysis with inclusion of gravity is aimed at eliciting errors in computing the amounts of heat transfer to and heating rates of the wetted/nose region of the ballistic missile at various altitudes and sea level for disregarding the effects of the gravitational 
force. It is observed that the gravitational effects are not always too small to be acceptable and most depend on the ballistic factor. On the whole these errors as found in the present analysis are greater in a light ballistic missile $\left(K_{B} \gg 1\right)$ than in a relatively heavy one $\left(K_{B} \ll 1\right)$ whereas the engineering trends are towards construction of the latter type of missile. Therefore Miele's (1962) analysis neglecting the gravitational effects is justified to some extent, if not fully. Further, what is baffling is that Miele (1962) obtained the velocity-altitude distribution of the missile, first without taking into account the effects of gravity and the second, taking them into account, but totally seting aside the effects of gravitational force in developing his theory of aerodynamic heating of ballistic missiles. One of the main reasons for this may be that Miele's (1962) main objective was to bring forth analytical solutions in closed form to the aerospace problems of ballistic missiles and as such he dispensed with the idea of gravity inclusion in his investigations of ballistic missile heating problems perhaps in apprehension of not getting relevant solutions in closed form. Finally it is worthwhile to enunciate that the present feature of aerodynamic heating problems does not account for any change in the velocity distribution espoused by variation in the flight-path inclination. Nevertheless, it is hoped that the errors appearing due to this discrepancy, though very small, will be dealt with in another paper.

\section{List of symbols}

$C \quad$ constant associated with the initial conditions;

$C_{D} \quad$ coefficient of missile drag;

$C_{F} \quad$ equivalent skin-friction coefficient;

$D \quad$ drag for the ballistic missile;

$\operatorname{Ei}(\pi)$

$g$

$h$

$K_{B}$

$K_{B F}$

$Q$

exponential integral $\int_{-\infty}^{\pi} e_{\pi}^{\pi} d \pi$;

acceleration due to gravity;

altitude of the missile with nondimensional altitude $\eta=h / \lambda$;

ballistic factor;

part of the ballistic factor, due to the friction drag;

$Q_{i}$ and final $Q_{f}$;

$Q_{1} \quad$ heat transferred to the wetted area without considering the effects of gravity with $Q_{2}$ as a part of it due to inclusion of the gravitational effects;

$Q_{R} \quad$ reference-heat energy associated with the wetted area;

$q \quad$ heat transfer at either stagnation or turbulent point of the nose region of the ballistic missile;

$S_{W} \quad$ the wetted area of the missile;

$u$ nondimensional velocity of the missile with initial and final values $u_{i}, u_{f}$ respectively;

$V \quad$ velocity of the missile with its initial value $V_{i}$ and final value $V_{f}$;

$W \quad$ the weight of the missile with its surface area $S$;

$x, y, z \quad$ index parameters concerned with rate $q$ of heat transfer with $C$ as the constant of variation; vide table 1 ;

air density, with its initial value $\rho_{0}$ at sea level;

$\theta \quad$ path inclination of the missile with respect to the horizon, with its initial value $\theta_{i}$; 
$\lambda \quad$ numerical constant $=23800 \mathrm{ft}=7254.4$ metres;

$\pi \quad$ nondimensional attitude parameter with its initial and final values $\pi_{t}, \pi_{f}$ respectively;

-(dot sign) derivative with respect to time $t$.

\section{References}

Miele A 1962 Flight mechanics (Reading, MA: Addison-Wesley) pp 308-323

Maitra S N 1979 Reentry of a ballistic missile into the earth's atmosphere for low ballistic factor $\left(K_{B} \leq 2\right)$. Indian J. Mech. Math. 17: 64-83

Summerfield M 1986 Tactical missile aerodynamics (New York: AIAA) vol. 104, pp 421-478 